\title{
MYCOPLASMA PULMONIS, AGENTE DA MICOPLASMOSE RESPIRATÓRIA MURINA: REVISÃO
}

\author{
(Mycoplasma pulmonis, Murine Respiratory Mycoplasmosis Agent: A Review) \\ Jenif Braga de Souza ${ }^{1}$, Mariana Thomaz de Oliveira, Elmiro Rosendo do \\ Nascimento, Maurício Afonso Verícimo, Maria Lúcia Barreto
}

${ }^{1}$ Correspondência: jenifbsouza@gmail.com

\begin{abstract}
RESUMO: A micoplasmose murina é uma infecção que ainda hoje representa um grande desafio para a experimentação animal em todo o mundo. O Mycoplasma pulmonis é o agente da micoplasmose respiratória murina, uma doença de curso crônico, lento e persistente por toda a vida do animal. O objetivo deste trabalho foi traçar um panorama sobre aspectos biológicos, a enfermidade, métodos de diagnóstico e as dificuldades de prevenção e controle deste microrganismo. Ratos e camundongos são usados em todo o mundo na pesquisa biológica, e quando possuem padronização sanitária aliada à padronização genética fornecem ao pesquisador modelos importantes para a compreensão de diversos mecanismos biológicos. Entretanto, essas espécies podem infectar-se por agentes patogênicos que, introduzidos na criação, afetam a produção e os resultados experimentais. Entre as principais infecções que acometem os roedores, a micoplasmose é uma das mais importantes porque produz problemas respiratórios e reprodutivos graves, comprometendo a criação e impedindo a obtenção de animais livres de patógenos. Mesmo com o advento de equipamentos como as estantes ventiladas e os microisoladores, a ocorrência da infecção ainda é 100\% nas colônias convencionais e 3,2\% a $20 \%$ em colônias mantidas sob barreiras.
\end{abstract}

Palavras-chave: Micoplamose Respiratória Murina; Mycoplama pulmonis; Rato de Laboratório; Camundongo de laboratório

ABSTRACT: Murine mycoplasmosis is an infection that still represents a challenge to animal testing throughout the world. Mycoplasma pulmonis is the agent of murine respiratory mycoplasmosis, a chronic course disease, slow and persistent throughout the animal's life. The objective of this paper was to give an overview on biological characteristics, disease, diagnostic methods and difficulties for prevention and control of this microorganism. Rats and mice are used worldwide in biological research, and when under sanitary and genetic standardization, provide the researcher important models for understanding of various biological mechanisms. However, these species can be infected by pathogens that, when introduced in the colony, affect production and experimental results. Among the major infections that affect rodents, mycoplasmosis is one of the most important because it produces severe respiratory and reproductive problems, affecting the colony and hindering the obtaining of pathogen-free animals. Even with the advent of equipment such as racks of individually ventilated cages, the occurrence of infection is still $100 \%$ in conventional colonies and $3.2 \%$ to $20 \%$ in colonies maintained under barriers.

Key Words: Micoplamose Respiratory Murine; Mycoplama pulmonis; Laboratory mouse; Laboratory rat. 


\section{INTRODUÇÃO}

O Mycoplasma pulmonis é o agente etiológico da micoplasmose respiratória murina (M.R.M.) que ainda hoje representa um grande desafio para a experimentação animal em todo o mundo. Mesmo com o advento de equipamentos como as estantes ventiladas e os microisoladores, a ocorrência da infecção ainda é 100\% nas colônias convencionais e $3,2 \%$ a $20 \%$ em colônias mantidas sobbarreiras (Barreto et al., 2002; Liang et al., 2009; Mähler e Köhl, 2009; Na et al., 2010; Schoondermark-van de Ven et al., 2006). O M. pulmonis produz uma queda significativa na produtividade da colônia, uma morbidade alta e nos estágios finais da doença um alto índice de mortalidade. Além disso, a micoplasmose causada por esse agente, em sua forma sub-clínica, pode produzir interferências nos resultados experimentais afetando significativamente os dados obtidos (Cassell et al., 1979, 1986; Cassell e Hill, 1979; Lindsey et al., 1971; Nicklas et al., 1999; Schoeb e McConnell, 2011; Steiner e Brown, 1993). Também foi relatada a presença desse agente em cultura celular prejudicando experimentos que utilizam este insumo (Harasawa et al., 1993; Mcgarrity et al., 1992). Outro aspecto importante é que a doença causada pelo $M$. pulmonis tem sido utilizado como modelo de referência para compreensão das micoplasmoses respiratórias humanas e animal (Davidson et al., 1981; HickmanDavis et al., 1997; Lindsey, Cassell e Davidson, 1982; Xu et al., 2006).

\section{DESENVOLVIMENTO}

\section{Histórico}

Em 1915, quando o uso de animais de laboratório foi intensificado, foram descritos os primeiros casos de broncopneumonia e infecção pulmonar em ratos (Cassell, 1982). O primeiro isolamento de micoplasma em ratos com doença pulmonar ocorreu em 1937 e em camundongos em 1940 (Beeuwkes e Collier, 1941; Klieneberger-Nobel, 1967; KlienebergerNobel e Steabben, 1937; Sabin, 1939; Sabin e Johnson, 1940).

Lutsky e Organick

(1966) verificaram pela primeira vez, que $\circ M$. pulmonis era capaz de produzir doença respiratória em camundongos convencionais e gnotobióticos. Situação semelhante foi observada por Kohn e Kirk (1969) ao inocular ratos gnotobióticos com $M$. pulmonis verificando a doença respiratória também nessa espécie. Posteriormente esses achados foram confirmados por 
outros pesquisadores (Jersey et al., 1973; Lindsey et al., 1971; Lindsey e Cassell, 1973; Whittlestone et al., 1972) e só foi possível pelo avanço da tecnologia empregada no desenvolvimento de animais gnotobióticos e SPF.

Em 1971, Lindsey et al. (1971), confirmaram que o $M$. pulmonis era 0 patógeno primário da doença crônica respiratória (DCR), sugerindo a mudança de nome para a Micoplasmose Pulmonar de Roedores e, em 1986 passam a denominar a doença de Micoplasmose Respiratória Murina (MRM) (Lindsey et al., 1986).

As espécies patogênicas de Mycoplasma isoladas com mais freqüência em ratos e camundongos são $M$. pulmonis, $M$. arthritidis, $M$. neuroliticum, $M$. collis, e $M$. muris (Davidson et al., 1994; Kaplan e Garvey, 1986; Kunita et al., 1989).

Em virtude da significância da presença do M. pulmonis nos biotérios, o objetivo deste trabalho foi traçar um panorama sobre aspectos biológicos, a enfermidade, métodos de diagnóstico e as dificuldades de prevenção e controle deste microrganismo.

\section{Distribuição e ocorrência}

O M. pulmonis é 0 agente etiológico da Micoplasmose Respiratória
Murina (MRM) e Micoplasmose genital e tem sido detectado em todo mundo (Barreto et al., 2002; Liang et al., 2009; Mähler e Köhl, 2009; Na et al., 2010; Schoondermark-van de Ven et al., 2006).

Schoondermark-van de Ven et al. (2006) utilizando soro de roedores enviados ao monitoramento sanitário de 2000 a 2003 na Europa ocidental encontrou que a ocorrência de $M$. pulmonis era de $3 \%$ para os ratos e $2 \%$ para camundongos, mesmo em colônias mentidas sobre barreiras. Da mesma forma, Mähler e Köhl, (2009) também utilizando soro de roedores enviados ao monitoramento sanitário encontram que a prevalência de $M$. pulmonis em ratos de 2007 a 2008 na Europa ocidental era de $3,8 \%$. Em um estudo realizado pelo Centro nacional de Animais de Laboratório em Taiwan, no período de 2004 até 2007 demonstrou que a ocorrência de $M$. pulmonis em ratos naquele país era de $40 \%$ para ratos e $20 \%$ para camundongos (Liang et al., 2009). Na et al. (2010) avaliaram as condições de camundongos SPF vendidos de centros comerciais de animais de laboratório na Coreia de 2006 a 2007 e encontraram a presença de M. pulmonis em 1,8 \% das amostras.

No Brasil, embora relatos da presença de micoplasmas em colônias 
de roedores já houvesse ocorrido (Timenetsky e Luca De, 1998), Barreto et al. (2002) tipificaram pelo uso da imunofluorescencia indireta $\circ M$. pulmonis em amostras obtidas de animais com sintomatologia respiratória e baixa performance reprodutiva, pela primeira vez no Rio de Janeiro. Ferreira et al. (2008) verificaram a presença de M. pulmonis em trabalhadores de biotérios, configurando-se como um agente zoonótico, com prováveis implicações na saúde pública.

\section{Transmissão e apresentação clinica}

O Mycoplasma pulmonis é o agente etiológico da MRM que é uma doença de caráter crônico, que se desenvolve lentamente, de forma silenciosa e é afetada por diferentes fatores que influenciam a relação parasito/hospedeiro, tais como: o gás cloro (Elmes e Bell, 1963), a amônia (Broderson et al., 1976; Lindsey et al., 1985; Schoeb et al., 1982), o vírus Sendai (Lindsey, 1986; Schoeb et al., 1985) as características genéticas do microorganismo e do hospedeiro (Cartner et al., 1996; Davidson et al., 1988; Davis et al., 1985; Davis e Cassell, 1982; Denison et al., 2005; Hickman-Davis et al., 1997; Love et al., 2010; Reyes et al., 2004).
A transmissão ocorre na forma vertical ou horizontal. Nelson (1948) relatou, pela primeira vez, a transmissão do micoplasma, por via respiratória de ratos para camundongos através de aerossóis. Posteriormente outros pesquisadores mostraram que $0 M$. pulmonis é transmitido por via uterina e que, ao invadir a placenta, atravessa a barreira placentária e infecta o líquido amniótico e os fetos (Burton et al., 2012; Cassell et al., 1979; Furr e TaylorRobinson, 1993; Love et al., 2010; Steiner et al., 1993). Burton et al. (2012) observou a presença de M. pulmonis no liquido aminiotico, vagina e endométrio de ratas inoculadas experimentalmente pela via hematogênica.

O M. pulmonis coloniza a mucosa epitelial do aparelho respiratório e reprodutor de ratos e camundongos (Cassell et al., 1981). Segundo Cassell (1982), a falta de sintomatologia, de correlação entre sinais clínicos e alterações patológicas e de uniformidade das lesões entre membros de mesma caixa e idade em uma colônia dificulta a detecção imediata da MRM. Quando uma colônia manifesta sinais clínicos, isso se deve à presença de animais em estágios terminais da doença (Banerjee et al., 1985; Brown e Reyes, 1991; Cassell et al., 1981, 1986; Cassell e Hill, 1979; Furr e Taylor- 
Robinson, 1993; Lindsey, 1986; Simecka et al., 1992).

Em sua forma clínica, os sintomas não são específicos, mas bastante sugestivos, caracterizando-se por espirros frequentes, estertores, postura arqueada, taquipnéia, secreção nasal e ocular, perda de peso acentuada, pêlo arrepiado, prurido cutâneo intenso, inclinação da cabeça por comprometido do ouvido médio e baixa fertilidade (Barreto et al., 2003; Davidson et al., 1994; Graham e Schoeb, 2011; Love et al., 2010; Reyes et al., 2004; Simecka et al., 1992). As lesões mais encontradas no aparelho respiratório são rinite aguda e crônica, otite média, laringite, traqueíte e broncopneumonia com bronquectasia e abscessos pulmonares em casos mais avançados (Cassell et al., 1979; Jersey, Whitehair e Carter, 1973; Kohn, 1971a; b; Lindsey et al., 1971; Lutsky e Organick, 1966).

Macroscopicamente, as lesões não são patognomônicas, porém sugestivas, caracterizando-se por secreções límpidas a purulentas encontradas no trato respiratório superior, no ouvido médio e/ ou no aparelho reprodutor (Perreau e Joubert, 1982). Segundo Jersey et al. (1973), o pulmão apresenta áreas vermelhas e/ou cinzas de consolidação (hepatização e atelectasia) que podem evoluir para bronquectasia contendo exudato mucopurulento ou caseopurulento (Barreto et al., 2003; Cassell, 1982; Jersey et al., 1973; Kohn, 1971b).

Histologicamente o exudato é constituído principalmente de neutrófilos e em todo aparelho respiratório observase infiltrado linfóide. $\mathrm{Na}$ traquéia ocorrem alterações no epitélio de revestimento e a perda dos cílios. No pulmão destaca-se a hiperplasia do tecido linfóide peribronquial (BALT), o infiltrado linfóide perivascular e a hiperplasia do epitélio dos brônquios associado a um exudato mucopurulento ocasionando a bronquectasia. Pode ocorrer engurgitamento dos capilares, edema e hemorragia nos espaços alveolares. $O$ intenso acúmulo de linfócitos em torno dos brônquios causa a atelectasia. Este quadro pode envolver um ou mais lóbulos (Barreto et al., 2003; Cassell et al., 1979; Henriksson et al., 2004; Hickman-Davis et al., 1997; Kohn, 1971b; Lutsky e Organick, 1966).

\section{Patogenia}

A adesão do micoplasma à superfície das células e sua posição extracelular foram descritas em camundongos e em ratos (Kohn, 1971b; Organick et al., 1966). Eventualmente, 
foi visualizado no interior das células epiteliais dos brônquios, mas sempre nos vacúolos como consequência do processo de endocitose (Kohn, 1971a).

Os micoplasmas expressam um grande número de lipoproteína exposta na superfície celular, sendo caracterizada como importante antígeno celular (Razin e Tully, 1995). Contudo, a lipoproteína e algumas proteínas integrais de membrana podem apresentar variabilidade antigênica, propriedade importante, para evadir a resposta imune do hospedeiro (Rottem, 2003; Vink et al., 2012). A lipoproteína produzida pelo o M. pulmonis é a VSA, constituída de uma região de 242 aminoácidos que possuem uma porção variável de carboxila terminal. $\mathrm{O}$ gene vsa é dividido em sete regiões que dependendo da sua expressão codificam as variantes VsaA, VsaC, VsaE, VsaF, VsaG, VsaH, e Vsal. A variação na expressão do gene vsa aparentemente ocorre devido o tempo de infecção, ao tropismo tecidual, passagens em meio de cultura $\mathrm{e}$ suscetibilidade do hospedeiro (Denison et al., 2005; Gumulak-Smith et al., 2001). Essa variação possui um papel fundamental na capacidade do $M$. pulmonis de evitar a resposta imune adaptativa e lise do complemento, modular a interação patógeno- hospedeiro,

exercer

atividade antifagocítica e formar biofilmes (Bolland et al., 2012; Bolland e Dybvig, 2012; Daubenspeck et al., 2009; Denison et al., 2005; Shaw et al., 2012; Simmons e Dybvig, 2007, 2009).

Algumas cepas de M. pulmonis expressam um exopolissacarídeo capsular, chamado de EPS-I polissacarídeo, que também é responsável pela adesão à célula hospedeira e redução da ligação do micoplasma com macrófagos alveolares, protegendo o microrganismo da fagocitose. A presença deste exopolissacarídeo sugere que existe outro caminho para o escape do $M$. pulmonis ao sistema imune (Bolland et al., 2012; Bolland e Dybvig, 2012; Daubenspeck et al., 2009; Shaw et al., 2013).

\section{Genoma}

Os micoplasmas comparados com outras bactérias possuem um pequeno genoma, cujo, a taxa de G-C é muito baixa (23 a $40 \mathrm{~mol} \%$ ) e por isso uma baixa capacidade de biossíntese (Davidson et al., 1994; Rosenbusch, 1994). O sequenciamento completo do genoma do $M$. pulmonis demonstrou que seu tamanho estava entre $964 \mathrm{pb}$, sendo um dos maiores genomas entre os Mollicutes, contendo 
aproximadamente $26,6 \%$ de $\mathrm{G}+\mathrm{C}$ e $73 \%$ de $A+T$, podendo variar entre cepas. As regiões que codificam as proteínas possuem entorno de $782 \mathrm{pb}$ e como fatores de virulência foi verificado a presença de genes que codificam hemolisina, nucleases, thiol peroxidase (Chambaud et al., 2001).

\section{Métodos de Diagnóstico de Micoplasmas}

Há um número limitado de testes úteis para a detecção de micoplasmas, que podem ser agrupados em categorias: isolamento em meios especificos, ELISA Indireto, histopatologia e PCR. A infecção subclínica é o maior desafio desses testes e a taxa de detecção é mais elevada e confiável quando métodos de diagnóstico são utilizados em conjunto (Cassell et al., 1984; Davidson et al., 1981, 1994; Harasawa et al., 1990).

O diagnóstico do $M$. pulmonis segue os mesmos preceitos dos demais micoplamas. Entretanto, em se tratando de micoplasmose murina, embora todos esses métodos possam ser utilizados, é necessário levar em consideração o padrão sanitário dos animais em teste e a prevalência da doença em colônias convencionais e colônias com barreiras (Cassell et al., 1984; Davidson et al., 1994).
Cultivo e isolamento

Em 1937, Klieneberger-Nobel e Steabben (1937), necessitaram de um meio enriquecido para conseguir isolar o microorganismo causador da pneumonia murina. O meio SP4 tem como base o meio de Edward (1947), mas foi desenvolvido por Tully et al. (1977), substituindo o soro equino pelo soro fetal bovino e adicionando um suplemento especial próprio para cultura de tecidos o CRML 1066 (Tully, 1987). Porém, Davidson et al. (1994) descreveram o meio A como ideal para $M$. pulmonis, $M$. arthritidis, $M$. neuroliticum e $M$. collis, reconhecendo que o meio SP4 para o $M$. muris é rotineiramente utilizado.

Diferentes sítios e métodos de coleta de material têm sido descritos e/ou comparados: swab e lavado do ouvido médio (Hill, 1971; Lentsch et al., 1979); swab da orofaringe (Davidson et al., 1981; Kohn, 1971b; Lentsch et al., 1979); impressão do tecido em ágar sólido (Kohn, 1971b); macerado dos tecidos (pulmão, traquéia, aparelho reprodutor) (Lentsch et al., 1979; TaylorRobinson et al., 1972). Diversos estudos posteriores recomendam que as amostras para cultivo sejam provenientes de sítios múltiplos (Cassell et al., 1984, 1986; Davidson et al., 1981, 
1994; FELASA,, 2014; LUSSIER et al., 1991; Simecka et al., 1992).

Amostras de tecidos ao serem maceradas devem ser diluídas como forma de impedir a ação de enzimas que possam inibir o crescimento, interferindo nos resultados (Cassell et al., 1984). Os cultivos em meio solido e liquido devem ser acompanhados por 21 dias. O M. pulmonis é fermentador de glicose e por isso durante o seu crescimento, a cor do meio se altera de vermelho para 0 amarelo, pela acidificação do mesmo (KARLAMANIS et al., 1969).

Uma vez isoladas e
caracterizadas as colônias de micoplasmas a tipificação da espécie é feita através de métodos sorológicos, como a inibição do crescimento, imunofluorescência

e a imunoperoxidase (Davidson et al., 1994; Goll, 1994; Nascimento et al., 2002).

\section{REAÇÃO EM CADEIA DA POLIMERASE}

A reação da cadeia da polimerase (PCR), aliado a cultura, é amplamente utilizada para o diagnóstico e identificação de espécies de micoplasmas (Buzinhani et al., 2007; Harasawa et al., 1990; Kuppeveld et al., 1992; Sanchez et al., 1994).
Harasawa et al.

(1990) examinaram pela primeira vez o uso do PCR na detecção do $M$. pulmonis e concluíram que o seu uso, associado a testes sorológicos, é valioso para monitorar as infecções por micoplasma. $E$, desde então variações desta técnica tem sido propostas, tais como utilização de sondas, Nested PCR, PCR-RFLP (Kim et al., 2005; Loganbill et al., 2005; Razin et al., 1987; Schoeb et al., 1997; Takahashi-Omoe et al., 2004). As técnicas de biologia molecular são capazes de detectar a presença de cepas de $M$. pulmonis não cultiváveis ou muito difíceis de cultivar em caldos. Essas cepas, não sendo detectáveis por métodos usuais, seriam responsáveis por falhas nos programas sanitários desenvolvidos em colônias com padrão sanitário definido e por comprometimento das pesquisas (Goto et al., 1994, 2012; Harasawa et al., 1990; Kunita et al., 1989; Kuppeveld et al., 1992, 1993).

\section{Detecção de antígeno em tecidos}

O exame anatomopatológico dos órgãos afetados fornece um diagnóstico presuntivo que deve ser confirmado por outros métodos de diagnóstico (Barreto et al., 2003; Lamb, 1975). As lesões são geralmente sugestivas (Simecka et al., 1992), uma vez que, outros 
microrganismos podem provocar lesões semelhantes (Cartner et al., 1996; Davidson et al., 1988; Davis et al., 1985; Davis e Cassell, 1982; Denison et al., 2005; Hickman-Davis et al., 1997; Love et al., 2010; Reyes et al., 2004; Schoeb et al., 1985). Além disso, as alterações microscópicas são mínimas ou ausentes nas primeiras semanas da infecção (Sanchez et al., 1994) e nas infecções crônicas as lesões microscópicas podem estar presentes mesmo não havendo alterações macroscópicas (Cassell, 1982).

A detecção de antígeno de micoplasma em tecido infectado pelos métodos de Imunofluorescência e Imunoperoxidase tem sido descrita por diferentes autores e são auxiliares ao diagnóstico, embora sejam dependentes da concentração de microorganismos presentes no tecido do hospedeiro (Cassell et al., 1983; Davidson et al., 1981, 1994; Freundt, 1981; Gourlay, 1981; Gourlay e Howard, 1983; Hill, 1983, 1978; Jordan, 1983; Lussier et al., 1991; Lutsky et al., 1986; Simecka et al., 1992).

\section{ELISA}

O ELISA é uma das técnicas mais empregadas no monitoramento sanitário de roedores de laboratório em razão de sua simplicidade, versatilidade, capacidade de testar várias amostras, velocidade e economia. É um teste barato, fácil e rápido, podendo ser feito sem grandes complexidades nos grandes laboratórios (Campos et al., 2000; Cassell et al., 1984; Cassell e Brown, 1983; FELASA, 2014; SánchesVizcaíno e Alvarez, 1987).

O ELISA indireto para detecção de anticorpos é o método de eleição para o diagnóstico da micoplasmose, pois não é necessário eutanasiar os animais como no caso de testes de cultura e histopatologia. É um ótimo teste de diagnóstico quando há quadro clínico (Davis et al., 1987), permitindo que vários animais sejam testados ao mesmo tempo (Barreto et al., 2002; Cassell e Brown, 1983; Davis et al., 1987; Sánches-Vizcaíno e Alvarez, 1987). No entanto, a técnica não permite a detecção em animais jovens, por não possuírem o seu sistema imune amadurecido (Cassell et al., 1984). Além disso, animais recém infectados podem levar até três meses para que seu sistema imune desenvolva anticorpos específicos (Cassell et al., 1984; Davis et al., 1987; Horowitz e Cassell, 1978; Sanchez et al., 1994; Watson et al., 1987).

Archives of Veterinary Science, v.21, n.4, p.08-25, 2016. 


\section{Prevenção e controle}

Ainda hoje o controle da presença do Mycoplasma pulmonis nas criações é difícil principalmente devido a múltiplas características do agente. No passado, algumas tentativas foram feitas para desenvolver uma vacina (Araujo et al., 2003; Barry et al., 1995; Cassell e Davis, 1978; Lai et al., 1990, 1991, 1994; Taylor et al., 1977) estes estudos não avançaram e ainda hoje não existem vacinas para prevenção da micoplasmose respiratória murina, provavelmente em virtude da grande variabilidade antigênica do microrganismo. Portanto, a prevenção ainda hoje basear-se na identificação do agente, seguida da eliminação dos animais infectados e prevenção de recontaminações por meio da interposição de barreiras sanitárias e medidas de biosegurança (Cassell et al., 1981; FELASA, 2014).

A histerectomia é um método utilizado na obtenção de animais livres de micoplasmas, porém a capacidade de atravessar a barreira placentária interfere no sucesso da técnica (Burton et al., 2012; Peltier et al., 2003, 2007; Peltier e Brown, 2005; Riggs et al., 2008; Steiner e Brown, 1993; Steiner et al., 1993). Para obtenção de melhores resultados recomenda-se a associação da histerectomia ao tratamento com antimicrobianos que interferem no metabolismo dos lipídeos, na síntese dos aminoácidos e dos ácidos nucleicos, no entanto, ainda assim é pouco efetivo e recomendado apenas para colônias convencionais (Banerjee et al., 1987; Bowden et al., 1994; Cassell et al., 1981).

As técnicas de limpeza de embriões associadas à reprodução assistida estão sendo as mais aconselhadas atualmente para eliminação de agentes patogênicos de colônias de roedores (Glenister e Thornton, 2000; Mansfield et al., 2010).

\section{CONCLUSÃO}

O M. pulmonis, agente da MRM, ainda hoje representa um grande desafio para biotérios de criação e experimentação em todo mundo. Mesmo em países aonde os biotérios possuem barreiras mais rígidas observa-se a persistência da ocorrência do agente (Mähler e Köhl, 2009).

Embora muitos estudos tenham sido desenvolvidos desde identificação deste agente, em 1937, para melhor compreensão da MRM, sua disseminação e seu controle. No entanto, ainda há muito a se elucidar sobre suas características biológicas, genotípicas, fenotípicas do M. pulmonis, como essas se relacionam com 0 
hospedeiro, para direcionamento de medidas mais eficazes de controle da MRM.

\section{REFERÊNCIAS}

ARAUJO, J. I.; TIMENETSKY, J.; ÁVILA, F. A. Avaliação da Imunogenicidade de uma Vacina Experimental Obtida a partir de Mycoplasma pulmonis Isolado de Ratos (Rattus norvegicus) Naturalmente Infectados. Ars Veterinaria, v. 19, n. 1, p. 80-86, 2003.

BANERJEE, A. K.; ANGULO, A. F.; POLAK-VOGELZANG, A. A. et al. Naturally occurring genital mycoplasmosis in mice. Laboratory Animals, v. 19, n. 4, p. 275-276, 1985. BANERJEE, A. K.; ANGULO, A. F.; POLAK-VOGELZANG, A. A. et al. An alternative method for the decontamination of rats carrying Mycoplasma pulmoni without the use of germfree isolators. Laboratory Animals, v. 21, n. 2, p. 138-142, 1987. BARRETO, M. L.; NASCIMENTO, E. R. DO; CAMPOS, C. A. M. et al. Detection of Mycoplasma pulmonis in laboratory rats. Brazilian Journal of Microbiology, v. 33, n. 3, p. 260-264, 2002.

BARRETO, M. L.; NASCIMENTO, E. R.; PRESGRAVE, R. et al. Diagnóstico patológico da micoplasmose respiratória murina em Rattus novergicus. Acta Scientiae Veterinariae, v. 31 , n. 2, p. 81-7, 2003.

BARRY, M. A.; LAI, W. C.; ALBERT JOHNSTON, S. Protection against mycoplasma infection using expressionlibrary immunization. Nature, v. 377, n. 6550, p. 632-635, 1995.

BEEUWKES, H.; COLLIER, W. A. Studies on arthrotropic pleuropneumonia like microorganisms. Journal of Infection Disease, v. 70, p. 1-6, 1941.

BOLLAND, J. R.; DYBVIG, K. Mycoplasma pulmonis Vsa proteins and polysaccharide modulate adherence to pulmonary epithelial cells. FEMS microbiology letters, v. $331, n$. $1, p$. 25-30, 2012.

BOLLAND, J. R.; SIMMONS, W. L.; DAUBENSPECK, J. M. et al. Mycoplasma polysaccharide protects against complement. Microbiology (Reading, England), v. 158, n. 7, p. 1867-1873, 2012.

BOWDEN, J. J.; SCHOEB, T. R.; LINDSEY, J. R. et al. Dexamethasone and oxytetracycline reverse the potentiation of neurogenic inflammation in airways of rats with Mycoplasma pulmonis infection. American Journal of Respiratory and Critical Care Medicine, v. 150, n. 5, p. 1391-1401, 1994.

BRODERSON, J. R.; LINDSEY, J. R.; CRAWFORD, J. E. The role of environmental ammonia in respiratory mycoplasmosis of rats. The American Journal of Pathology, v. 85, n. 1, p. 115-130, 1976.

BROWN, M. B.; REYES, L. Immunoglobulin class- and subclassspecific responses to Mycoplasma pulmonis in sera and secretions of naturally infected Sprague-Dawley female rats. Infection and Immunity, $v$. 59, n. 6, p. 2181-2185, 1991.

BURTON, A.; KIZHNER, O.; BROWN, M. B. et al. Effect of experimental genital mycoplasmosis on gene expression in the fetal brain. Journal of Reproductive Immunology, v. 93, n. 1, p. 9-16, 2012.

BUZINHANI, M.; METIFFOGO, E.; TIMENETSKY, J. Detection of Mycoplasma spp. and Ureaplasma diversum in cown with reproductive disorders. Arquivo Brasileiro de Medicina Veterinária e Zootecnia, v. 59, n. 6, p. 1368-1375, 2007. Acesso em: $14 / 12 / 2014$.

CAMPOS, C. DE M.; NASCIMENTO, E. DO; BARRETO, M. L. et al. ELISA for the diagnosis of infections by Mycoplasma pulmonis and Mycoplasma 
arthritidis in laboratory rats (Rattus norvegicus). Revista Brasileira de Medicina Veterinária, v. 22, n. 5, p. 207-210, 2000.

CARTNER, S. C.; SIMECKA, J. W.; BRILES, D. E. et al. Resistance to mycoplasmal lung disease in mice is a complex genetic trait. Infection and Immunity, v. 64, n. 12, p. 5326-5331, 1996.

CASSELL, G. H. The pathogenic potential of mycoplasmas: Mycoplasma pulmonis as a model. Reviews of Infectious Diseases, v. 4, p. 18-34, 1982.

CASSELL, G. H.; BROWN, M. B. [1983]. Enzyme-linked Immunosorbent Assay (ELISA) for Detection of Antimycoplasmal Antibody. Methods in Mycoplasmology. v. 1, p.457-469, 1983. Disponível em: <http://www.sciencedirect.com/science/a rticle/pii/B9780125838016500767>.

Acesso em: 16/12/2014.

CASSELL, G. H.; DAVIS, J. K. Protective effect of vaccination against Mycoplasma pulmonis respiratory disease in rats. Infection and Immunity, v. 21, n. 1, p. 69-75, 1978.

CASSELL, G. H.; DAVIS, J. K.; COX, N. R. et al. Mycoplasma pulmonis detection in rodents: lessons for diagnosis in other species. Israel Journal of Medical Sciences, v. 20, n. 9, p. 859-865, 1984. CASSELL, G. H.; DAVIS, J. K.; SIMECKA, J. W. et al. Mycoplasmal infections: Disease pathogenesis, implications for biomedical research and control. . In: BHATT, P. N., JACOBY, R. O., MORSE III, H. C. \& NEW, A. E. Viral and mycoplasmal infections of laboratory rodents.1.ed. New York: Academic Press,1986, Cap. V, p.87130.

CASSELL, G. H.; HILL, A. Murine \& other small-animal Mycoplasmas. In: BARILE, M. F., RAZIN, S., TULLY, J. G. \& WHITCOMB, R. F. The Mycoplasmas.1.ed. New York: Academic Press, 1979, p.235-273.
CASSELL, G. H.; LINDSEY, J. R.; BAKER, H. J.; DAVIS, J. K. Mycoplasmal and rickettsial diseases. In: BAKER, H. J., LINDSEY, J. R. \& WEISBROTH, S. H. The Laboratory Rat. 1.ed. New York: Academic Press, 1979, p.243-269.

CASSELL, G. H.; LINDSEY, J. R.; DAVIS, J. K. Respiratory and genital mycoplasmosis of laboratory rodents: implications for biomedical research. Israel Journal of Medical Sciences, v. 17, n. 7, p. 548-554, 1981.

CASSELL, G. H.; WILBORN, W. H.; SILVERS, S. H. et al. Adherence and colonization of Mycoplasma pulmonis to genital epithelium and spermatozoa in rats. Israel Journal of Medical Sciences, v. 17, n. 7, p. 593-598, 1981. CHAMBAUD, I.; HEILIG, R.; FERRIS, $\mathrm{S}$; et al. The complete genome sequence of the murine respiratory pathogen Mycoplasma pulmonis. Nucleic Acids Research, v. 29, n. 10, p. 2145-2153, 2001.

DAUBENSPECK, J. M.; BOLLAND, J. R.; LUO, W. et al. Identification of exopolysaccharide-deficient mutants of Mycoplasma pulmonis. Molecular Microbiology, v. 72 , n. 5, p. 12351245, 2009.

DAVIDSON, M. K.; DAVIS, J. K.; LINDSEY, J. R. et al. Clearance of different strains of Mycoplasma pulmonis from the respiratory tract of $\mathrm{C} 3 \mathrm{H} / \mathrm{HeN}$ mice. Infection and Immunity, v. 56, n. 8, p. 2163-2168, 1988.

DAVIDSON, M. K.; GAMBILL, G. P.; CASSELL, G. H. et al. Mycoplasmas of Laboratory Rodents. In: Whitford H. W.; Rosenbusch R. F.; Lauerman L. H.; Mycoplasmosis in Animals: Laboratory Diagnosis. 1. ed. Ames: Wiley-Blackwell, 1994, Cap. 4, p.39-50. DAVIDSON, M. K.; LINDSEY, J. R.; BROWN, M. B. et al. Comparison of methods for detection of Mycoplasma pulmonis in experimentally and naturally infected rats. Journal of Clinical 
Microbiology, v. 14, n. 6, p. 646-655, 1981.

DAVIS, J.; CASSELL, G. H.; GAMBILL, $G$. et al. Diagnosis of murine mycoplasmal infections by enzymelinked immunosorbent assay (ELISA). Israel Journal of Medical Sciences, v. 23, n. 6, p. 717-722, 1987.

DAVIS, J. K.; CASSELL, G. H. Murine respiratory mycoplasmosis in LEW and F344 rats: strain differences in lesion severity. Veterinary Pathology, v. 19, n. 3, p. 280-293, 1982.

DAVIS, J. K.; PARKER, R. F.; WHITE, $\mathrm{H}$. et al. Strain differences in susceptibility to murine respiratory mycoplasmosis in C57BL/6 and $\mathrm{C} 3 \mathrm{H} / \mathrm{HeN}$ mice. Infection and Immunity, v. 50, n. 3, p. 647-654, 1985. DENISON, A. M.; CLAPPER, B.; DYBVIG, K. Avoidance of the host immune system through phase variation in Mycoplasma pulmonis. Infection and Immunity, v. 73, n. 4, p. 2033-2039, 2005.

EDWARD, D. G. F. A selective medium for pleuropneumonia-like organisms. Journal of General Microbiology, v. 1, n. 2, p. 238-243, 1947.

ELMES, P. C.; BELL, D. The Effects of Chlorine Gas on the Lungs of Rats with Spontaneous Pulmonary Disease. The Journal of Pathology and Bacteriology, v. 86, p. 317-327, 1963. FEDERATION OF LABORATORY ANIMAL SCIENCE ASSOCIATIONS FELASA, WORKING GROUP ON REVISION OF GUIDELINES FOR HEALTH MONITORING OF RODENTS AND RABBITS; MÄHLER, C. M.; BERARD, M. et al. FELASA recommendations for the health monitoring of mouse, rat, hamster, guinea pig and rabbit colonies in breeding and experimental units. Laboratory Animals, v. 48, n. 3, p. 178-192, 2014.

FERREIRA, J. B.; YAMAGUTI, M.; MARQUES, L. M. et al. Detection of Mycoplasma pulmonis in laboratory rats and technicians. Zoonoses and Public Health, v. 55, n. 5, p. 229-234, 2008.

FURR, P. M.; TAYLOR-ROBINSON, D. Factors influencing the ability of different mycoplasmas to colonize the genital tract of hormone-treated female mice. International Journal of Experimental Pathology, v. 74, n. 1, p. 97-101, 1993. GLENISTER, P. H.; THORNTON, C. E. Cryoconservation--archiving for the future. Mammalian Genome, v. 11, n. 7, p. 565-571, 2000.

GOLL, F. Identification of Mycoplasmas isolated from domestic animals. In: WHITTFORD, H. W.; ROSENBUSCH, R. F.; LAUERMAN, L. $H$. Mycoplasmosis in animals: Laboratory diagnosis. 1.ed. lowa: lowa State University Press, Ames, 1994, Cap. 3, p. 15-31.

GOTO, K.; KUNITA, S.; TERADA, E. et al. Comparison of polymerase chain reaction and culture methods for detection of Mycoplasma pulmonis from nasal, tracheal and oral swab samples of rats. Experimental Animals, v. 43, n. 3, p. 413-415, 1994.

GOTO, K.; YAMAMOTO, M.; ASAHARA, M. et al. Rapid Identification of Mycoplasma pulmonis Isolated from Laboratory Mice and Rats Using MatrixAssisted Laser Desorption Ionization Time-of-Flight Mass Spectrometry. Journal of Veterinary Medical Science, v. 74 , n. 8, p. 1083-1086, 2012.

GRAHAM, J. E.; SCHOEB, T. R. Mycoplasma pulmonis in Rats. Journal of Exotic Pet Medicine, v. 20, n. 4, p. 270-276, 2011.

GUMULAK-SMITH, J.; TEACHMAN, A.; $\mathrm{TU}, \mathrm{A} . \mathrm{H}$. T. et al. Variations in the surface proteins and restriction enzyme systems of Mycoplasma pulmonis in the respiratory tract of infected rats. Molecular Microbiology, v. 40, n. 4, p. 1037-1044, 2001.

HARASAWA, R.; KOSHIMIZU, K.; UEMORI, T. et al. The polymerase chain reaction for Mycoplasma pulmonis. 
Microbiology and Immunology, v. 34, n. 4, p. 393-395, 1990.

HARASAWA, R.; MIZUSAWA, H.; NOZAWA, $\mathrm{K}$. et al. Detection and tentative identification of dominant mycoplasma species in cell cultures by restriction analysis of the 16S-23S rRNA intergenic spacer regions. Research in Microbiology, v. 144 , n. 6, p. 489-493, 1993.

HENRIKSSON, G.; HELGELAND, L.; MIDTVEDT, T. et al. Immune response to Mycoplasma pulmonis in nasal mucosa is modulated by the normal microbiota. American Journal of Respiratory Cell and Molecular Biology, v. 31, n. 6, p. 657-662, 2004. HICKMAN-DAVIS, J. M.; MICHALEK, S. M.; GIBBS-ERWIN, J. et al. Depletion of alveolar macrophages exacerbates respiratory mycoplasmosis in mycoplasma-resistant C57BL mice but not mycoplasma-susceptible $\mathrm{C} 3 \mathrm{H}$ mice. Infection and immunity, v. 65, n. 6, p. 2278-2282, 1997.

HILL, A. Mycoplasma caviae, a new species. Journal of General Microbiology, v. 65 , n. 1, p. 109-113, 1971.

HOROWITZ, S. A.; CASSELL, G. H. Detection of antibodies to Mycoplasma pulmonis by an enzyme-linked immunosorbent assay. Infection and Immunity, v. 22, n. 1, p. 161-170, 1978. JERSEY, G. C.; WHITEHAIR, C. K.; CARTER, G. R. Mycoplasma pulmonis as the primary cause of chronic respiratory disease in rats. Journal of the American Veterinary Medical Association, v. 163, n. 6, p. 599-604, 1973.

KAPLAN, P. J.; GARVEY, J. S. Mycoplasma neurolyticum membranes: a $\mathrm{T}$-independent antigen in the rat. Immunological Investigations, v. 15, n. 1, p. 35-53, 1986.

KARLAMANIS, E.; THOMAS, L.; KASTAVROPOULOS, $\mathrm{K}$. et al. Mycoplasmicidal action of normal tissue extracts. , v. 221, p. 860-861, 1969.
KIM, D.-J.; PARK, J.-H.; SEOK, S.-H. et al. Differential identification of mycoplasma pulmonis and $\mathrm{M}$. arthritidis using PCR-based RFLP. Experimental Animals / Japanese Association for Laboratory Animal Science, v. 54, n. 4, p. 359-362, 2005.

KLIENEBERGER-NOBEL,

E. Mycoplasma, a brief historical review. Annals of the New York Academy of Sciences, v. 143, n. 1, p. 713-718, 1967.

KLIENEBERGER-NOBEL; STEABBEN, D. B. On a pleuropneumonia - like organism in lung lesions of rats with notes on the clinical and pathological features of the underlying codition. Journal of Hygiene, v. 37, p. 143-152, 1937.

$\mathrm{KOHN}$, D. F. Bronchiectasis in rats infected with Mycoplasma pulmonis: an electron microscopy study. Laboratory Animal Science, v. 21, n. 6, p. 856861, 1971a.

KOHN, D. F. Sequential pathogenicity of Mycoplasma pulmonis in laboratory rats. Laboratory Animal Science, v. 21, n. 6, p. 849-855, 1971b.

KOHN, D. F.; KIRK, B. E. Pathogenicity of Mycoplasma pulmonis in laboratory rats. Laboratory Animal Care, v. 19, n. 3, p. 321-330, 1969.

KUNITA, S.; TERADA, E.; GHODA, A. et al. Genomic DNA analysis of rodent mycoplasmas. Jikken Dobutsu. Experimental Animals, v. 38, n. 3, p. 201-206, 1989.

KUPPEVELD, F. J. VAN; LOGT, J. T. VAN DER, A. F. et al. Genus- and species-specific identification of mycoplasmas by 16S rRNA amplification. Applied and Environmental Microbiology, v. 58, n. 8, p. 2606-2615, 1992.

KUPPEVELD, F. J. VAN; MELCHERS, W. J.; WILLEMSE, H. F.; et al. Detection of Mycoplasma pulmonis in experimentally infected laboratory rats by $16 \mathrm{~S}$ rRNA amplification. Journal of 
Clinical Microbiology, v. 31, n. 3, p. 524-527, 1993.

LAI, W. C.; BENNETT, M.; GORDON, B. $\mathrm{E}$. et al. Protection of mice against experimental murine mycoplasmosis by a Mycoplasma pulmonis immunogen in lysogenized Escherichia coli. Vaccine, v. 12, n. 4, p. 291-298, 1994.

LAI, W. C.; BENNETT, M.; LU, Y. S. et al. Biological evaluation of Mycoplasma pulmonis temperature-sensitive mutants for use as possible rodent vaccines. Infection and Immunity, v. 58, n. 7, p. 2289-2296, 1990.

LAI, W. C.; BENNETT, M.; LU, Y. S. et al. Vaccination of Lewis rats with temperature-sensitive mutants of Mycoplasma pulmonis: adoptive transfer of immunity by spleen cells but not by sera. Infection and Immunity, v. 59, n. 1, p. 346-350, 1991.

LENTSCH, R. H.; WAGNER, J. E.; OWENS, D. R. Comparison of techniques for primary isolation of respiratory Mycoplasma pulmonis from rats. Infection and Immunity, v. 26, n. 2, p. 590-593, 1979.

LIANG, C.-T.; SHIH, A.; CHANG, Y.-H. et al. Microbial contaminations of laboratory mice and rats in Taiwan from 2004 to 2007. Journal of the American Association for Laboratory Animal Science: JAALAS, v. 48, n. 4, p. 381386, 2009.

LINDSEY, J. R. Prevalence of viral and mycoplasmal infections in laboratory rodents. In: BHATT, P. N., JACOBY, R. O., MORSE III, H. C. \& NEW, A. E. Viral and Mycoplasmal Infections of Laboratory Rodents: Effects on Biomedical Research. 1.ed. New York: Academic Press, 1986, Cap.X, p.801808.

LINDSEY, J. R.; BAKER, H. J.; OVERCASH, R. G. et al. Murine chronic respiratory disease. Significance as a research complication and experimental production with Mycoplasma pulmonis. The American Journal of Pathology, v. 64 , n. 3, p. 675-708, 1971.
LINDSEY, J. R.; CASSELL, G. H. Experimental Mycoplasma pulmonis Infection in Pathogen-Free Mice. The American Journal of Pathology, v. 72, n. 1, p. 63-90, 1973.

LINDSEY, J. R.; CASSELL, G. H.; BACKER, H. J. Diseases due to mycoplasmas and Rickettsias. In: BERNIRSCHKE, K., GARNER, F. M. \& JONES, T. C. Pathology of laboratory animals. 2.ed. New York: Spring Verlag, 1986, p.1481-1517.

LINDSEY, J. R.; CASSELL, G. H.; DAVIDSON, M. K. Mycoplasmal and other bacterial diseases of the respiratory system. In: FOSTER, H. L., FOX J. G. The mouse in biomedical research. The mouse in biomedical research. 1.ed. New York: Academic Press, 1982, p.21-41.

LINDSEY, J. R.; DAVIDSON, M. K.; SCHOEB, T. R. et al. Mycoplasma pulmonis-host relationships in a breeding colony of Sprague-Dawley rats with enzootic murine respiratory mycoplasmosis. Laboratory Animal Science, v. 35, n. 6, p. 597-608, 1985. LOGANBILL, J. K.; WAGNER, A. M.; BESSELSEN, D. G. Detection of Mycoplasma pulmonis by Fluorogenic Nuclease Polymerase Chain Reaction Analysis. Comparative Medicine, v. 55, n. 5, p. 419-424, 2005.

LOVE, W.; DOBBS, N.; TABOR, L. et al. Toll-like receptor 2 (TLR2) plays a major role in innate resistance in the lung against murine Mycoplasma. PloS One, v. 5, n. 5, p. e10739, 2010.

LUSSIER, G.; DAVIS, J. K.; SHEK, W. R. et al. Detection methods for the identification of rodent viral and mycoplasmal infections. Subcommittee on Rodent Viral and Mycoplasmal Infections of the American Committee on Laboratory Animal Disease (ACLAD). Laboratory Animal Science, v. 41, n. 3, p. 199-225, 1991.

LUTSKY, I. I.; ORGANICK, A. B. Pneumonia due to mycoplasma in gnotobiotic mice. I. Pathogenicity of 
Mycoplasma pneumoniae, Mycoplasma salivarium, and Mycoplasma pulmonis for the lungs of conventional and gnotobiotic mice. Journal of Bacteriology, v. 92, n. 4, p. 1154-1163, 1966.

MÄHLER, M.; KÖHL, W. A serological survey to evaluate contemporary prevalence of viral agents and Mycoplasma pulmonis in laboratory mice and rats in western Europe. Lab Animal, v. 38, n. 5, p. 161-165, 2009. MANSFIELD, K. G.; RILEY, L. K.; KENT, M. L. Workshop Summary: Detection, Impact, and Control of Specific Pathogens in Animal Resource Facilities. ILAR Journal, v. 51, n. 2, p. 171-179, 2010.

MCGARRITY, G. J.; KOTANI, H.; BUTLER, G. H. Mycoplasmas and tissue culture cells. In: MANILOFF, J.; MCELHANEY R. N.; FINCH, L. R.; BASEMAN, J. B. Mycoplasmas: Molecular Biology and Pathogenesis. 1.ed. Washington, DC: Am Soc Microbiol, 1992,Cap. IX p. 445-454.

NASCIMENTO, E. R.; BARRETO, M. L.; PLATENIK, M. O. et al. Contagious Agalactia by Mycoplasma agalactiae in goats in Brazil: Etiologic study. In: XIV CONGRESS OF THE INTERNATIONAL ORGANIZATION FOR MYCOPLASMOLOGY, 2002, Vienna. Anais..., Vienna: International Organization for Mycoplasmology, 2002. p.421.

NA, Y.-R.; SEOK, S.-H.; LEE, H.-Y. et al. Microbiological quality assessment of laboratory mice in Korea and recommendations for quality improvement. Experimental Animals / Japanese Association for Laboratory Animal Science, v. 59, n. 1, p. 25-33, 2010.

NELSON, J. B. The nasal transmission of pleuropneumonia-like organisms in mice and rats. The Journal of Infectious Diseases, v. 82, n. 2, p. 169-176, 1948.
NICKLAS, W.; HOMBERGER, F. R.; ILLGEN-WILCKE, B. et al. Implications of infectious agents on results of animal experiments: Report of the Working Group on Hygiene of the Gesellschaft für Versuchstierkunde-Society for Laboratory Animal Science (GVSOLAS). Laboratory Animals, v. 33, n. suppl 1, p. 39-87, 1999.

ORGANICK, A. B.; SIEGESMUND, K. A.; LUTSKY, I. I. Pneumonia due to Mycoplasma in Gnotobiotic Mice II. Localization of Mycoplasma pulmonis in the Lungs of Infected Gnotobiotic Mice by Electron Microscopy. Journal of Bacteriology, v. 92, n. 4, p. 1164-1176, 1966.

PELTIER, M. R.; BARNEY, B. M.; BROWN, M. B. Effect of experimental genital mycoplasmosis on production of matrix metalloproteinases in membranes and amniotic fluid of Sprague-Dawley rats. American Journal of Reproductive Immunology, v. 57, n. 2, p. 116-121, 2007.

PELTIER, M. R.; BROWN, M. B. Experimental genital mycoplasmosis causes increased levels of mRNA for IL6 and TNF-alpha in the placenta. American Journal of Reproductive Immunology, v. 53, n. 4, p. 189-198, 2005.

PELTIER, M. R.; RICHEY, L. J.; BROWN, M. B. Placental lesions caused by experimental infection of SpragueDawley rats with Mycoplasma pulmonis. American Journal of Reproductive Immunology, v. 50, n. 3, p. 254-262, 2003.

PERREAU, P.; JOUBERT, L. Les mycoplasmoses animales. Revue de medecine veterinaire, v. 133 , p. 539 552, 1982.

RAZIN, S.; HYMAN, H. C.; NUR, I. et al. DNA probes for detection and identification of mycoplasmas (Mollicutes). Israel Journal of Medical Sciences, v. 23, n. 6, p. 735-741, 1987. RAZIN, S.; TULLY, J. G. Molecular and Diagnostic Procedures in 
Mycoplasmology, Volume 1:
Molecular Characterization. San
Diego: Academic Press, 1995. 473 p.
REYES, L.; SHELTON, M.; RIGGS, M.
et al. Rat strains differ in susceptibility to
maternal and fetal infection with
Mycoplasma pulmonis. American
Journal of Reproductive Immunology,
v. 51, n. 3, p. 211-219, 2004. RIGGS, M. A.; MAUNSELL, F. P.; REYES, L. et al. Hematogenous infection of Sprague-Dawley rats with Mycoplasma pulmonis: development of a model for maternal and fetal infection. American Journal of Obstetrics \& Gynecology, v. 198, n. 3, p. 318.e1318.e7, 2008.

ROSENBUSCH, R. F. Biology and taxonomy of the mycoplasmas. In: Whitford, H. W.; Rosenbusch, R. F.; Lauerman, L. H. Mycoplasmosis in Animals: Laboratory Diagnosis. 1. ed. Ames: Wiley-Blackwell, 1994, Cap. 1, p.3-11.

ROTTEM, S. Interaction of Mycoplasmas With Host Cells. Physiological Reviews, v. 83, n. 2, p. 417-432, 2003.

SABIN, A. B. Mice as Carriers of Pathogenic Pleuropneumonia-like Microorganisms. Science (New York, N.Y.), v. 90, n. 2323, p. 18-19, 1939.

SABIN, A. B.; JOHNSON, B. Pathogenic pleuropnemonia- like microorganisms in tissues of normal mice and isolation of new immunological types. Proceedings of the Society of Experimental Biology Medicine, v. 44, p. 569-571, 1940.

SÁNCHES-VIZCAÍNO, J. M.; ALVAREZ, $M$. C. Enzyme Immunoassay Techniques, ELISA, in Animal and Plant Diseases. Technical Series 7, OIE Office International des Epizooties, Instituto Nacional de Investigaciones Agrarias, n. 2, p. 54, 1987.

SANCHEZ, S.; TYLER, K.; ROZENGURT, N. et al. Comparison of a PCR-based diagnostic assay for Mycoplasma pulmonis with traditional detection techniques. Laboratory Animals, v. 28, n. 3, p. 249-256, 1994. SCHOEB, T. R.; DAVIDSON, M. K.; LINDSEY, J. R. Intracage ammonia promotes growth of Mycoplasma pulmonis in the respiratory tract of rats. Infection and Immunity, v. 38, n. 1, p. 212-217, 1982.

SCHOEB, T. R.; DYBVIG, K.; KEISLING, K. F. et al. Detection of Mycoplasma pulmonis in cilia-associated respiratory bacillus isolates and in respiratory tracts of rats by nested PCR. Journal of Clinical Microbiology, $v$. 35, n. 7, p. 1667-1670, 1997.

SCHOEB, T. R.; KERVIN, K. C.; LINDSEY, J. R. Exacerbation of Murine Respiratory Mycoplasmosis in Gnotobiotic F344/N Rats by Sendai Virus Infection. Veterinary Pathology Online, v. 22, n. 3, p. 272-282, 1985. SCHOEB, T. R.; MCCONNELL, E. E. Commentary: further comments on Mycoplasma pulmonis and lymphoma in bioassays of rats. Veterinary Pathology, v. 48, n. 2, p. 420-426, 2011.

SCHOONDERMARK-VAN DE VEN, E. M. E.; PHILIPSE-BERGMANN, I. M. A.; VAN DER LOGT, J. T. M. Prevalence of naturally occurring viral infections, Mycoplasma pulmonis and Clostridium piliforme in laboratory rodents in Western Europe screened from 2000 to 2003. Laboratory Animals, v. 40 , n. 2 , p. 137-143, 2006.

SHAW, B. M.; DAUBENSPECK, J. M.; SIMMONS, W. L. et al. EPS-I polysaccharide protects Mycoplasma pulmonis from phagocytosis. FEMS microbiology letters, v. 338 , n. 2, p. 155-160, 2013.

SHAW, B. M.; SIMMONS, W. L.; DYBVIG, K. The Vsa shield of Mycoplasma pulmonis is antiphagocytic. Infection and Immunity, v. 80, n. 2, p. 704-709, 2012.

SIMECKA, J. W.; DAVIS, J. K.; DAVIDSON, M. K. et al. Mycoplasma diseases of animals. In: Maniloff, J.; 
McElhaney, R.; Finch, L.; Baseman, J. Mycoplasmas: Molecular Biology and Pathogenesis. 1.ed. New York: ASM Press, 1992, Cap x, p.391-411.

SIMMONS, W. L.; DYBVIG, K. Biofilms protect Mycoplasma pulmonis cells from lytic effects of complement and gramicidin. Infection and Immunity, v. 75, n. 8, p. 3696-3699, 2007.

SIMMONS, W. L.; DYBVIG, K. Mycoplasma biofilms ex vivo and in vivo.

FEMS Microbiology Letters, v. 295, n. 1, p. 77-81, 2009.

STEINER, D. A.; BROWN, M. B. Impact of experimental genital mycoplasmosis on pregnancy outcome in SpragueDawley rats. Infection and Immunity, v. 61, n. 2, p. 633-639, 1993.

STEINER, D. A.; UHL, E. W.; BROWN, $M$. B. In utero transmission of Mycoplasma pulmonis in experimentally infected Sprague-Dawley rats. Infection and Immunity, v. 61 , n. 7 , p. 29852990, 1993.

TAKAHASHI-OMOE, H.; OMOE, K.; MATSUSHITA, $\mathrm{S}$. et al. Polymerase chain reaction with a primer pair in the 16S-23S rRNA spacer region for detection of Mycoplasma pulmonis in clinical isolates. Comparative Immunology, Microbiology and Infectious Diseases, v. 27, n. 2, p. 117-128, 2004.

TAYLOR, G.; HOWARD, C. J.; GOURLAY, R. N. Protective Effect of Vaccines on Mycoplasma pulmonisInduced Respiratory Disease of Mice. Infection and Immunity, v. 16, n. 2, p. 422-431, 1977.

TAYLOR-ROBINSON, D.; DENNY, F. W.; THOMPSON, G. W. et al. Isolation of mycoplasmas from lungs by a perfusion technique. Medical Microbiology and Immunology, v. 158, n. 1, p. 9-15, 1972.

TIMENETSKY, J.; DE LUCA, R. R. Detection of Mycoplasma pulmonis from rats and mice of São Paulo/SP, Brazil. Laboratory Animal Science, v. 48, n. 2, p. 210-213, 1998.
TULLY, J. G. Biology of Rodents mycoplasmas. In: BHATT, P. N., JACOBY, R. O., MORSE III, H. C. \& NEW, A. E. Viral and Mycoplasmal Infections of Laboratory Rodents: Effects on Biomedical Research. 1.ed. New York: Academic Press, 1987, Cap. $\mathrm{xX}$, p.63-88.

TULLY, J. G.; WHITCOMB, R. F.; CLARK, $H$. $F$. et al. Pathogenic mycoplasmas: cultivation and vertebrate pathogenicity of a new spiroplasma. Science (New York, N.Y.), v. 195, n. 4281, p. 892-894, 1977.

VINK, C.; RUDENKO, G.; SEIFERT, H. $S$. Microbial antigenic variation mediated by homologous DNA recombination. FEMS microbiology reviews, v. 36, $\mathrm{n}$. 5, p. 917-948, 2012.

WATSON, H. L.; COX, N. R.; DAVIDSON, M. K. et al. Mycoplasma pulmonis proteins common to murine mycoplasmas. Israel Journal of Medical Sciences, v. 23, n. 5, p. 442447, 1987.

WHITTLESTONE, P.; LEMCKE, R. M.; OLDS, R. J. Respiratory disease in a colony of rats. The Journal of Hygiene, v. 70, n. 3, p. 387-407, 1972.

$\mathrm{XU}, \mathrm{X}$; ZHANG, D.; ZHANG, $\mathrm{H}$. et al. Neutrophil histamine contributes to inflammation in mycoplasma pneumonia. The Journal of Experimental Medicine, v. 203, n. 13, p. 2907-2917, 2006. 